\title{
Caracterización de los pacientes con disfonía evaluados en la Unidad de Voz Pediátrica del Hospital Dr. Luis Calvo Mackenna
}

\section{Characterization of patients with dysphonia evaluated in the pediatric voice unit of the Dr. Luis Calvo Mackenna Hospital}

\author{
Diana Centeno A' 1 , Maximiliano Penna $\mathbf{R}^{2}$.
}

\begin{abstract}
RESUMEN
Introducción: La importancia del diagnóstico y manejo adecuado de los trastornos vocales en pacientes pediátricos, radica en que un mismo síntoma como la disfonía, puede tener etiologías de significación variable.

objetivo: Conocer la epidemiología y características de los pacientes pediátricos con disfonía crónica derivados a la Unidad de Voz del Hospital Dr. Luis Calvo Mackenna, y con ello generar un protocolo de diagnóstico y manejo coordinado entre fonoaudiólogos y otorrinolaringólogos.

Material y método: Estudio prospectivo descriptivo con revisión de fichas clínicas y protocolos operatorios de pacientes que se controlan en la Unidad de Voz del Hospital Dr. Luis Calvo Mackenna.

Resultados: En la unidad de voz hay en seguimiento 22 niños con una edad promedio de 10 años. Las lesiones diagnosticadas con mayor frecuencia mediante videonasolaringoscopía fueron los nódulos vocales $(n=10)$ y el aumento de volumen asimétrico a nivel cordal $(n=8)$. Se pudo realizar telelaringoscopía con videoestroboscopía en el 81,8\% de los pacientes. Del total de pacientes en control, 10 requirieron laringoscopía directa.

Conclusión: La disfonía en el niño suele ser un síntoma subestimado y, por ende, no tratado. Consideramos que una unidad de voz infantil debe preocuparse de evaluar sus pacientes con tecnología acorde a su edad y de realizar un seguimiento adecuado de los tratamientos.
\end{abstract}

Palabras clave: Voz, disfonía infantil, unidad de voz.

\begin{abstract} significance.
\end{abstract}

Introduction: Adequate diagnosis and proper management of voice disorders in pediatric patients lies in that the symptom dysphonia, can have etiologies of variable

Aim: To evaluate the epidemiology and characteristics of pediatric patients with chronic dysphonia that are referred to the voice unit of Dr. Luis Calvo Mackenna Hospi-

\footnotetext{
1 Departamento de Otorrinolaringología, Hospital Dr. Luis Calvo Mackenna, Santiago, Chile.

2 Departamento de Otorrinolaringología, Hospital del Salvador, Universidad de Chile, Santiago, Chile.

* Los autores declaran no tener conflictos de interés.
}

Recibido el 27 de marzo, 2018. Aceptado el 22 de julio, 2018. 
tal, and thereby generate a diagnostic protocol and coordinated management strategy between speech pathologist and otolaryngologist.

Material and method: Prospective descriptive study with review of clinical files and operative protocols of patients followed-up at the voice unit of the Dr. Luis Calvo Mackenna Hospital.

Results: In the voice unit, there are 22 children being followed up. The most frequently diagnosed lesions with videonasolaryngoscopy were vocal nodules $(n=10)$ and asymmetric volume on the vocal cord $(n=8)$. We performed telelaryngoscopy with videostroboscopy in $81.8 \%$ of patients. Of all the patients, 10 required direct laryngoscopy.

Conclusions: Dysphonia in children is usually an underestimated symptom and, therefore, not treated. We believe that a children's voice unit should evaluate their patients with the appropriate technology according to the patients age, and to carry out an adequate follow-up of the treatments administered.

Key words: Voice, pediatric dysphonia, voice unit.

\section{INTRODUCCIÓN}

La voz es uno de los medios principales para la expresión y comunicación, y en los niños es una herramienta importante para su desarrollo psicosocial, por lo que es de gran importancia dar a conocer su correcto uso y cuidado con el objetivo de promover la detección temprana de sus alteraciones y prevenirlas ${ }^{1,2}$. Los trastornos de la voz de más de 15 días de evolución 0 disfonías crónicas, deben ser evaluados por el otorrinolaringólogo. En el caso de los adultos, estas patologías se encuentran ampliamente descritas en cuanto a su etiología y diagnóstico, con tratamientos bastante bien protocolizados en su mayoría ${ }^{3}$. A diferencia de lo que ocurre en pediatría, en gran parte de los servicios 0 departamentos de otorrinolaringología de adultos, existen unidades dedicadas exclusivamente a la voz y sus patologías. En los últimos años, la tendencia a constituir unidades de voz pediátricas ha ido aumentando, lo que permite evaluar más detalladamente las disfonías en este grupo etario y sus probables tratamientos ${ }^{4}$.

La prevalencia de los desórdenes de la voz ha sido reportada entre $6 \%-23 \%$ en la población escolar según las distintas publicaciones ${ }^{1,5}$. En la mayoría de los casos, el problema de la voz no es percibido como una preocupación por los padres o los propios niños, sino que es referido por un individuo externo (profesor, familiar, etc.) lo que retrasa muchas veces el diagnóstico y el tratamiento oportuno $0^{6}$. La importancia del diagnóstico y manejo adecuado de los trastornos vocales en pacientes pediátricos radica en que un mismo síntoma como la disfonía, puede tener etiologías de significación variable, desde condiciones benignas como nódulos vocales hasta problemas complejos de la vía aérea como por ejemplo, la papilomatosis laríngea ${ }^{4,7}$. La videoestroboscopía es el método exploratorio de elección ya que permite observar detalladamente tanto la anatomía de las cuerdas vocales como la función fonatoria (apertura y cierre, vibración cordal, etc.) de las mismas ${ }^{8}$. En cuanto a la laringoscopía directa, es un procedimiento que puede tener utilidad diagnóstica y terapéutica simultáneamente. Se realiza en pabellón bajo sedación 0 anestesia general y permite la visualización y palpación de las anomalías estructurales de las cuerdas vocales. Su indicación es para aquellos pacientes en los que no podemos realizar los otros exámenes antes mencionados, cuando no hay respuesta a la terapia fonoaudiológica o cuando existe duda diagnóstica9 ${ }^{9}$.

En cuanto al manejo de las patologías vocales en los niños, éste se basa principalmente en la terapia vocal para lo cual es muy importante considerar el entorno social, familiar y emocional del paciente ${ }^{10}$. Solo en casos seleccionados, se requerirá un abordaje quirúrgico el cual, en niños, debe ser lo más conservador posible ${ }^{6,8}$.

\section{OBJETIVO}

El objetivo es conocer la epidemiología y características de los pacientes pediátricos con 
disfonía crónica que son derivados a la Unidad de Voz del Hospital Dr. Luis Calvo Mackenna, y con ello generar un protocolo de diagnóstico y manejo integral coordinado entre fonoaudiólogos y otorrinolaringólogos.

\section{MATERIAL Y MÉTODO}

Se realizó un estudio prospectivo descriptivo con revisión de fichas clínicas y protocolos operatorios de pacientes en seguimiento en la Unidad de Voz del Hospital Dr. Luis Calvo Mackenna, desde octubre de 2016 hasta marzo de 2018. La muestra corresponde a la población menor de 15 años con disfonía de más de 4 semanas de evolución, derivados de las zonas pertenecientes a la red asistencial del sector oriente de Santiago. Se excluyen en este estudio los pacientes con diagnóstico de papilomatosis laríngea ya que son evaluados y controlados por la unidad de vía aérea del hospital. Este trabajo fue aceptado por el Comité de Ética del Servicio de Salud Metropolitano Oriente y aprobado para su desarrollo. Los medios técnicos con los que cuenta la unidad de voz son: nasofibroscopio, videonasolaringoscopio, telelaringoscopios de $70^{\circ}$ y $90^{\circ}$, videoestroboscopía, laringoscopía directa y un programa gratuito de análisis acústico llamado Praat. Dentro de los recursos físicos contamos con tres salas: sala 1 de atención fonoaudiológica, sala
2 de atención otorrinolaringológica y sala 3 para realización de los procedimientos. Y en cuanto a recursos humanos: 1 otorrinolaringólogo, 1 fonoaudiólogo, 1 técnico de enfermería de nivel superior, 1 residente de otorrinolaringología, internos de fonoaudiología y 1 psicóloga infantil.

\section{RESULTADOS}

En la unidad de voz hay en seguimiento 22 niños con una edad promedio de 10 años (rango entre 4 y 15 años) cuya derivación proviene en su mayoría (46\%) de la atención primaria de salud, seguido de derivaciones por otorrinolaringólogos del mismo servicio de otorrinolaringología (Figura 1).

La distribución por género es $91 \%(\mathrm{n}=20)$ masculina y $9 \%(n=2)$ femenina. Las lesiones diagnosticadas con mayor frecuencia mediante videonasolarigoscopía y telelaringoscopía con videoestroboscopía, fueron los nódulos vocales y el aumento de volumen asimétrico a nivel cordal (Figura 2), arrojando un total de 23 lesiones (Tabla 1).

De los 22 pacientes, se pudo realizar telelaringoscopía con videoestroboscopía en 18 de ellos; el porcentaje restante que no fue sometido a este examen, se debió a dificultades técnicas por la edad que presentaban los pacientes al momento de estudio (4-5 años). El promedio de edad de los pacientes a los que se les realizó videoestro-

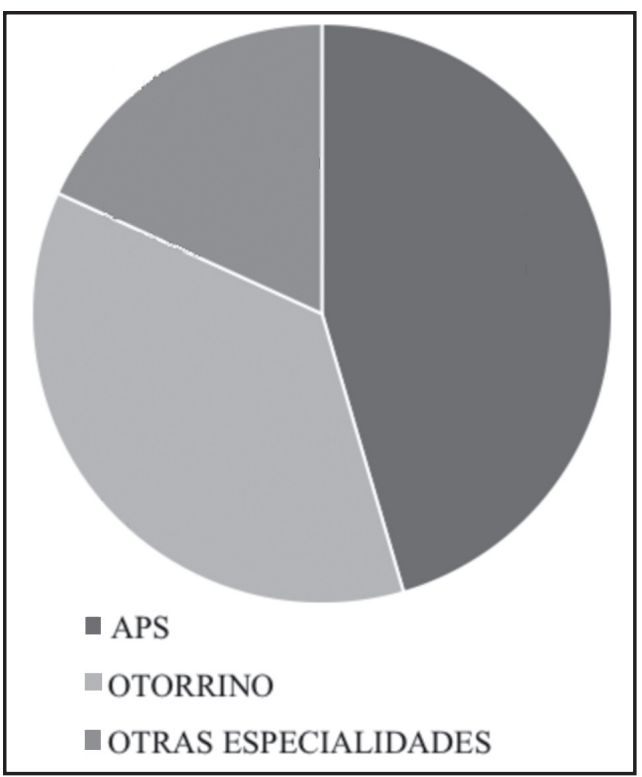

Figura 1. Procedencia de las derivaciones a la Unidad de Voz del Hospital Dr. Luis Calvo Mackenna. APS: Atención primaria salud. Otras especialidades: pediatría, broncopulmonar, servicio de urgencia. 


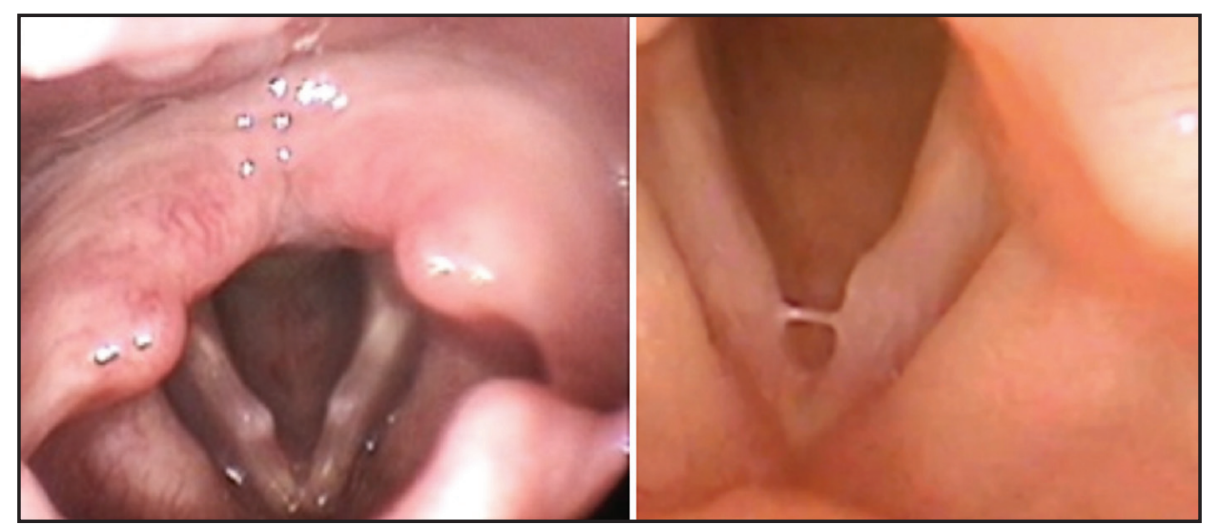

Figura 2. Panel izquierdo: paciente masculino de 12 años de edad derivado desde la atención primaria de salud a la Unidad de Voz del Hospital Dr. Luis Calvo Mackenna con diagnóstico de disfonía crónica. Mediante videonasolaringoscopía se realizó el diagnóstico de nódulos vocales. El paciente se encuentra en tratamiento fonoaudiológico. Panel derecho: paciente masculino de 12 años de edad derivado a la Unidad de Voz por médico tratante especialista en broncopulmonar del Hospital Dr. Luis Calvo Mackenna con diagnóstico de disfonía crónica. Mediante videonasolaringoscopía se objetivó un aumento de volumen asimétrico en ambas cuerdas vocales. Bajo laringoscopía directa se diagnosticó la presencia de una masa fibrosa subepitelial en cuerda vocal Izquierda y lesión por contragolpe en cuerda vocal derecha. Se realizó microcirugía laríngea para su resolución.

Tabla 1. Causas de disfonía infantil crónica en 22 pacientes diagnosticados mediante videonasolaringoscopía y telelaringoscopía con videoestroboscopía de la Unidad de Voz del Hospital Dr. Luis Calvo Mackenna

\begin{tabular}{|lc|}
\hline Diagnóstico & $\begin{array}{c}\text { Videonasolarigoscopía } \\
\text { Telelaringoscopía/Videoestroboscopía }\end{array}$ \\
\hline Nódulos vocales & 10 \\
Aumento volumen asimétrico & 8 \\
Sulcus vocal & 1 \\
Parálisis cordal & 1 \\
Pólipo cuerda vocal & 1 \\
Membrana laríngea anterior & 1 \\
Vasculodisgenesia & 1 \\
\hline
\end{tabular}

boscopía es de 10 años (7-14 años). Del total de pacientes en seguimiento en la unidad de voz, 10 de ellos requirieron laringoscopía directa debido a que no se pudo realizar exámenes diagnósticos o el diagnóstico era dudoso con los métodos exploratorios antes mencionados (4 pacientes), porque no presentaron evolución favorable con la terapia fonoaudiológica (5 pacientes) o porque presentaron diagnóstico con indicación quirúrgica en la primera evaluación (1 paciente). Las lesiones más frecuentemente encontradas fueron sulcus tipo I-II $(n=5)$ y pseudoquistes (masa fibrosa subepitelial) $(n=5)$.
El $45 \%$ del total de los pacientes, requirió de una evaluación diagnóstica con videonasolaringoscopía, telelaringoscopía con videoestroboscopía y laringoscopía directa. La utilidad de esta triple intervención radicó en lograr definir la etiología específica de la disfonía.

\section{DISCUSIÓN}

Existen varios estudios que se enfocan en los desórdenes vocales en niños sobre todo cuando 
existe disfonía crónica. A nivel nacional, las publicaciones disponibles son escasas. En una publicación de Olavarría y col se observó que, en 48 pacientes pediátricos con disfonía crónica, el diagnóstico posoperatorio más prevalente fue sulcus tipo II, seguido de quiste submucoso unilateral. La mayoría de estos pacientes habían sido catalogados inicialmente como portadores de nódulos vocales y reevaluados en unidad de voz por persistencia de síntomas a pesar de terapia fonoaudiológica ${ }^{9}$. De forma similar en nuestros pacientes, mediante la triple intervención (videonasolaringoscopía, telelaringoscopía con videoestroboscopía y laringoscopía directa), Iogramos redefinir la patología cordal como se ilustró anteriormente.

Connelly y cols, observaron en 137 niños mayores de 8 años con disfonía crónica, que el hallazgo más común fue el abuso vocal, representando el $45 \%$ (62 pacientes). De estos niños, 36 (58\%) tenían diagnóstico de nódulos vocales confirmado por nasofibroscopía o laringoscopía bajo visión micoscópica ${ }^{7}$. Así mismo, García Martins y cols en su estudio de desórdenes en la voz, observaron en pacientes menores de 18 años, que entre las lesiones laríngeas predominaron los nódulos vocales, con una incidencia del $41 \%$ en niños menores de 6 años, $73 \%$ entre los 7 y 12 años y $51 \%$ entre los 13 y 18 años lo que confirma la mayor incidencia de esta patología en la edad escolar ${ }^{5,3}$. En cuanto a la distribución por género, los nódulos vocales fueron más frecuentes en el sexo masculino hasta los 12 años. A partir de esta edad, los nódulos vocales fueron más frecuentes en niñas y mujeres adultas ${ }^{3}$.

El uso excesivo de la voz (sobreuso) y la voz de alta intensidad con esfuerzo (mal uso vocal), común en niños, contribuyen a la fisiopatología de los nódulos vocales. En tales condiciones, la aducción de los pliegues vocales tiene un impacto intenso, pudiendo causar trauma a nivel de la unión del tercio anterior con los dos tercios posteriores de las cuerdas vocales ${ }^{10,11}$. En consecuencia, pueden aparecer edemas, lesiones microvasculares y engrosamiento localizado bilateral del epitelio cordal a nivel del tercio medio. Después de la adolescencia, especialmente en varones, los nódulos vocales podrían tener una tendencia a mejorar espontáneamente. Por esta razón, la cirugía de los nódulos en los niños no es el tratamiento de elección, sino más bien la terapia vocal' ${ }^{12}$.

En cuanto a la evaluación de los niños con trastornos de la voz, la tendencia actual es que sean atendidos en clínicas especializadas de voz pediátrica las cuales ofrecen el beneficio de la examinación simultánea por un otorrinolaringólogo y un fonoaudiólogo. Además, estas unidades cuentan con equipamiento diagnóstico específico como videonasolaringoscopios, telelaringoscopios de $70^{\circ}-90^{\circ}$, estroboscopios flexibles y/o rígidos y laboratorio de v0z. La telelaringoscopía con videoestroboscopía requiere un mayor nivel de cooperación del niño, por lo que se utiliza como técnica de elección en los mayores de 6 años ${ }^{6}$. En nuestra casuística, el paciente de menor edad que fue sometido a telelaringoscopía con videoestroboscopía presentó 7 años de edad. Debido a esta complejidad técnica, en nuestra unidad adoptamos el término de "videoestroboscopía condicionada" que consiste en entregarles información al paciente y al tutor acerca del procedimiento y realizarla en las etapas necesarias hasta lograr la mejor visualización diagnóstica posible.

Para evaluar inicialmente la voz del paciente y el resultado de la terapia vocal y de la fonocirugía, en los adultos está ampliamente extendido el uso del Voice Handicap Index (VHI) como herramienta de valoración. Esta herramienta se ha adaptado para la edad pediátrica y ha sido validada al español junto a la versión abreviada ${ }^{13}$. El Pediatric Voice Handicap Index es una prueba confiable, que la completan los padres o tutores de niños con disfonía. Se caracteriza por su fácil aplicación en la práctica clínica diaria y brinda información adicional de cómo los niños perciben la disfonía en el ámbito funcional, físico y emocional. Se recomienda su uso e inclusión en los protocolos para evaluar la voz en la población pediátrica².

Smillie y cols muestran la experiencia de la clínica de voz pediátrica y aluden que los médicos generales fueron la principal fuente de referencia, habiendo también derivaciones significativas de las subespecialidades y de los mismos otorrinolaringólogos. En sus hallazgos, los nódulos de cuerda vocal representaron el $52 \%$ de los diagnósticos, y hubo un predominio masculino dentro de esta categoría de diagnóstico ${ }^{4}$. De manera similar, en nuestra investigación observamos que la mayoría de los pacientes ingresados a la unidad de voz, proviene de derivacio- 
nes de atención primaria de salud que efectúan los médicos generales, y además concuerda en que los hallazgos más frecuentes son los nódulos vocales. Algunos rasgos de personalidad se han asociado al desarrollo de nódulos vocales, como un comportamiento agresivo, conductas hiperactivas, inmadurez y dificultades en las relaciones sociales; además se ha observado que los niños con nódulos de cuerda vocal se caracterizan por realizar actos impulsivos, ser más distraídos, tener malas relaciones interpersonales y comportamientos inmaduros en comparación con compañeros sin nódulos ${ }^{14-16}$.

Landa y cols aconsejan la realización de rehabilitación vocal en todos los niños con disfonía crónica como primer abordaje terapéutico, considerando que trabajaron con menores entre $9 \mathrm{y}$ 16 años, los que colaborarían más en la terapia de rehabilitación. Ellos abogan que es de gran importancia para obtener buenos resultados, la implicancia de la familia en este proceso terapéutico. En relación al tratamiento quirúrgico, ellos lo reservan para aquellos casos en los que se sospecha la existencia de lesiones congénitas (quiste epidermoide, sulcus, puente mucoso, etc.) o aquellos que no mejoran con la foniatría ${ }^{8}$. Nosotros trabajamos de manera semejante a lo descrito por Landa y cols. La disfonía en el niño suele ser un síntoma subestimado y, por ende, no tratado. La razón principal es porque se asume que se trata de una condición benigna asociada al mal uso 0 abuso vocal. Si bien esto puede ser cierto en la mayoría de los casos, debe realizarse un esfuerzo en establecer un diagnóstico preciso y luego tratar la patología correspondiente ${ }^{9}$. Nuestra metodología de trabajo busca resolver esta problemática y sigue un flujograma de trabajo donde la evaluación inicial realizada al paciente es similar a la propuesta por la Sociedad Europea de Laringología ${ }^{17}$ (Figura 3).

En un estudio realizado por Rodríguez-Parra y cols se concluyó que sólo 38\% de los servicios de otorrinolaringología dispone de una unidad de voz, además que menos de la mitad de los servicios utilizan algún tipo de protocolo escrito en la evaluación de pacientes disfónicos, sin embargo, existe unanimidad en considerar necesario un protocolo de evaluación y tratamiento de la voz sistematizado $^{18}$. La unidad de voz debe formar parte del normal funcionamiento de un servicio de otorrinolaringología, estando implicada en labores organizativas, asistenciales, docentes y de investigación.

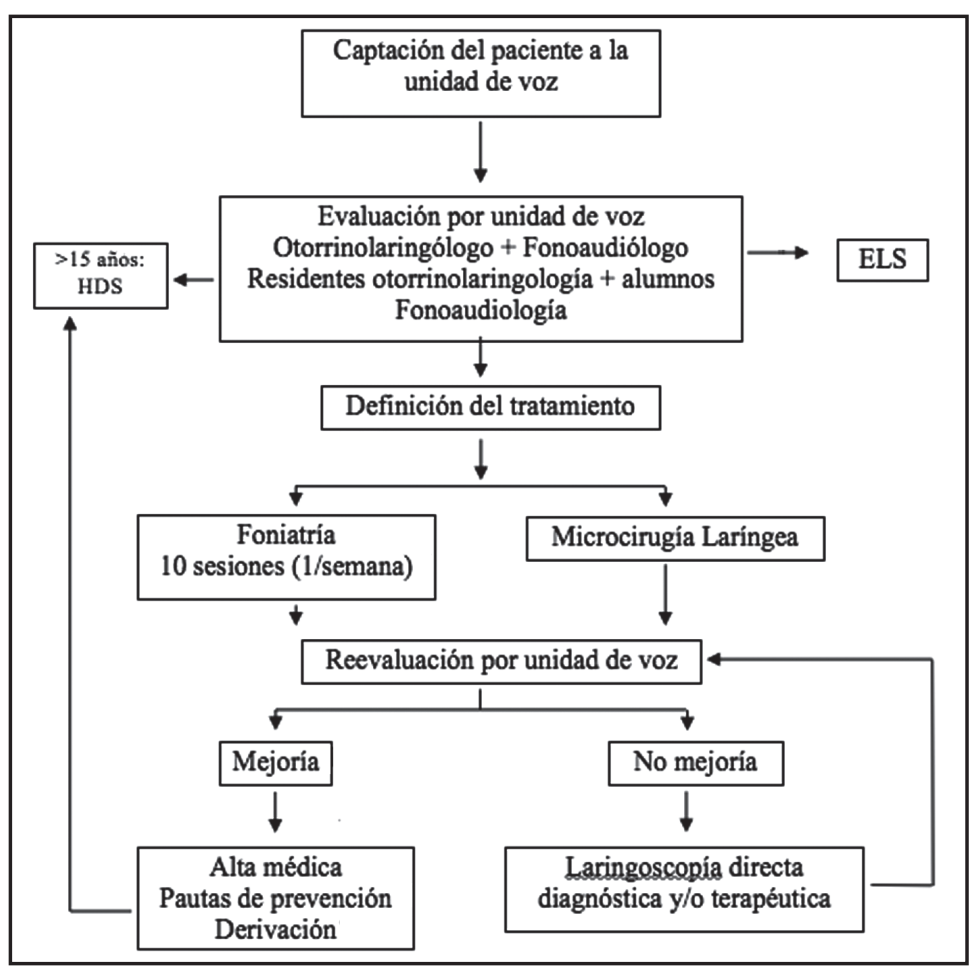

Figura 3. Flujograma diagnóstico y terapéutico que utilizamos en la Unidad de Voz Hospital Dr. Luis Calvo Mackenna. HDS: Hospital del Salvador. ELS: European Laryngological Society. 
Fundamental es que esté compuesta por personal calificado e interdisciplinar, un equipamiento mínimo (siendo imprescindible la estroboscopía) y un modo de actuación y criterios de derivación claramente definidos ${ }^{19}$. A nivel chileno, de acuerdo a nuestro conocimiento, la Unidad de Voz del Hospital Dr. Luis Calvo Mackenna sería la única unidad de voz exclusivamente pediátrica del país.

\section{BIBLIOGRAFÍA}

1. Hirschberg J, Dejonckere PH, Hirano M, Mori K, Schultz Coulon HJ, Vrticka K. Voice disorders in children. Int J Pediatr Otorhinolaryngol 1995; 32 Suppl: S109-25.

2. Sanz L, Bau P, Arribas I, Rivera T. Adaptation and validation of Spanish version of the pediatric Voice Handicap Index (P-VHI). Int J Pediatric Otorhinolaryngol 2015; 79: 1439-43.

3. Martins RH, Do Amaral ha, Tavares El, Martins MG, Goncalves TM, Dias NH. Voice Disorders: Etiology and Diagnosis. J Voice 2016; 30: 761. e1-761.e9.

4. Smillie I, McManus K, Cohen W, Lawson E, Wynne DM. The paediatric voice clinic. Arch Dis Child 2014; 99: 912-5.

5. Carding PN, Roulstone S, Northstone K. The Prevalence of Childhood Dysphonia : A Cross Sectional Study. J Voice 2006; 20: 623-30.

6. Possamai V, Hartley B. Voice disorders in children. Pediatr Clin North Am 2013; 60: 879-92.

7. Connelly A, Clement WA, Kubba H. Management of dysphonia in children. J Laryngol Otol 2009; 123: 642-7.

8. Landa M, Palicio I, Álvarez L, Martinez Z. Revisión de nuestra experiencia en fonocirugía infantil. Acta Otorrinolaringol Esp 2017; 68: 269-73.

9. Olavarría C, Cortez P. Evaluación y diagnóstico de la disfonía en niños. Neumol Pediatr 2014; 9: 75-9.

10. Tuzuner A, Demirci $S$, Oguz H, Ozcan KM. Pediatric Vocal Fold Nodule Etiology: What Are Its Usual Causes in Children? J Voice 2017; 31: 506.e19506.e23.

\section{CONCLUSIÓN}

Consideramos que una unidad de voz infantil debe preocuparse de evaluar a sus pacientes con la tecnología acorde a su edad y de realizar un seguimiento adecuado de los tratamientos indicados. Para esto, es fundamental el trabajo en conjunto entre otorrinolaringólogos y fonoaudiólogos.

11. WoHL DL. Nonsurgical management of pediatric vocal fold nodules. Arch Otolaryngol Head Neck Surg 2005; 131: 68-70.

12. Nardone H, Recko T, Huang L, Nuss R. A Retrospective Review of the Progression of Pediatric Vocal Fold Nodules. JAMA Otolaryngol Head Neck Surg 2014; 140: 233-6.

13. Sanz L, Bau P, Arribas I, Rivera T. Development and validation of a short version of the Spanish pediatric voice handicap index (P-VHI-10). Int J Pediatr Otorhinolaryngol 2016; 88: 113-6.

14. Garcia-Real T, Diaz-Roman T, Garcia-Martinez V, VIEIRO-IGLESIAS P. Clinical and acoustic vocal profile in children with attention deficit hyperactivity disorder. J Voice 2013; 27: 787.e11-787.e18

15. KAY NJ. Vocal nodules in children: a etiology and management. J Laryngol Otol 1982; 96: 731-6.

16. Green G. Psycho-behavioral characteristics of children with vocal nodules: WPBIC ratings. $J$ Speech Hear Disord 1989; 54: 306-12.

17. Friedrich G, Dejonckere PH. The voice evaluation protocol of the European Laryngological Society (ELS); first results of a multicenter study. Laryngorhinootologie 2005; 84: 744-52.

18. Rodriguez-Parra M, Casado J, Adrián J, Buiza J. Estado actual de los Servicios ORL españoles. Heterogeneidad en el manejo de los problemas de voz. Acta Otorrinolaringol Esp 2006; 57: 109-14.

19. Casado-Morente JC, Casanova-Castillo R, Hernández-Rubiño A y col. Procedimientos para la integración de una Unidad de Voz en el funcionamiento de un Servicio/Área de ORL y sus resultados. Acta Otorrinolaringol Esp 2011; 62: 454-61.

Correspondencia: Diana M. Centeno A

Hospital Dr. Luis Calvo Mackenna, Santiago, Chile

E mail: doctora@dianacentenootorrino.cl 